\title{
Brain Imaging in Stroke: Insight Beyond Diagnosis
}

\author{
May Nour • David S. Liebeskind
}

Published online: 10 May 2011

(C) The American Society for Experimental NeuroTherapeutics, Inc. 2011

\begin{abstract}
Stroke, whether hemorrhagic or ischemic in nature, has the ability to lead to devastating and debilitating patient outcomes, which not only has direct implications from a healthcare standpoint, but its effects are longstanding and they impact the community as a whole. For decades, the goal of advancement and refinement in imaging modalities has been to develop the most precise, convenient, widely available and reproducible interpretable modality for the detection of stroke, not only in its hyperacute phase, but a method to be able to predict its evolution through the natural course of disease. Diagnosis is one of the most important initial roles, which imaging fulfills after the identification of existent pathology. However, imaging fulfills an even more important goal by using a combination of imaging modalities and their precise interpretation, which lends itself to understanding the mechanisms and pathophysiology of underlying disease, and therefore guides therapeutic decision-making in a patienttailored fashion. This review explores the most commonly used brain imaging modalities, computer tomography, and magnetic resonance imaging, with an aim to demonstrate their dynamic use in uncovering stroke mechanism, facilitating prognostication, and potentially guiding therapy.
\end{abstract}

Keywords Ischemic stroke - Intracerebral hemorrhage · Computed tomography $\cdot$ Magnetic resonance imaging . Diffusion-weighted imaging $\cdot$ Perfusion-weighted imaging

Electronic supplementary material The online version of this article (doi:10.1007/s13311-011-0046-0) contains supplementary material, which is available to authorized users.

M. Nour • D. S. Liebeskind $(\bowtie)$

University of California at Los Angeles Stroke Center,

UCLA Medical Center,

Los Angeles, CA 90095, USA

e-mail: davidliebeskind@yahoo.com

\section{Introduction}

The importance of imaging in stroke extends far beyond its role in diagnosis to extend to one that is truly dynamic, lending insight into the underlying mechanisms, as well as guiding decision-making with respect to therapeutic intervention. The advancements in imaging techniques and refinement of complementary imaging modalities to best provide the most efficient, precise, cost-effective, convenient, and reproducible interpretation of data continues to be an ongoing quest. The advent of multimodal imaging in both computed tomography (CT) and magnetic resonance imaging (MRI) has provided a global perspective on cerebrovascular pathophysiology at the onset of stroke and throughout subsequent stroke evolution, allowing for a potent means of understanding stroke pathophysiology, whether hemorrhagic or ischemic in nature. Having reliable insight into the etiology of the disease is of utmost benefit to patients as therapeutic strategies are then tailored to the presentation, risk factors, and dynamic nature of the patient's disease progression. In this review, we will focus on the most commonly used brain imaging modalities, namely $\mathrm{CT}$ and MRI. The angiographic subsets of these modalities will be discussed in the next chapter with the understanding that both brain parenchymal, as well as vascular imaging, are integral for appreciating the full scope of these disorders.

\section{Commonly Used Imaging Modalities}

Noncontrast CT was once one of the only options for firstline assessment of stroke, and initially it had its benefit in being widely available in the community setting and in large stroke centers alike, as well as being fast and cost 
effective. With the advent of MRI and now the use of multimodal CT and MRI, including their respective angiographic subsets, the field of neuroimaging, and stroke in particular was transformed.In an acute stroke setting, multimodal CT typically includes noncontrast head CT, CT angiogram of the head and neck (CTA), and CT perfusion (Fig. 1). MRI sequences include diffusion-weighted imaging (DWI), perfusion-weighted imaging, T1, T2, fluidattenuated inversion recovery, gradient recalled echocardiogram (GRE), as well as MR angiography of the head and neck (Fig. 2). Therefore, multimodal CT and MRI not only capture the integrity of brain vasculature and parenchyma, but also offer crucial information in regard to cerebral perfusion and allowing for dynamic assessment of tissue viability.Therefore, the choice between multimodal $\mathrm{CT}$ and MRI is made on a patient by patient basis, for instance preferring CT in patients for whom MRI is contraindicated, or who are less medically stable, or for patients who are unable to remain supine for an extended period of time. Many institutions have developed tailored multimodal CT protocols that are of particular benefit in the setting of hyperacute, acute, subacute, and chronic stroke. Studies have detailed the protocol for multimodal CT to answer the following questions in a presentation of stroke: 1) Is there hemorrhage? 2) Is there occlusion of a proximal artery or intravascular thrombus that can be targeted for thrombolysis? 3) Is there a core of critically ischemic irreversibly infarcted tissue, and 4) Is there a penumbra of ischemic but potentially salvageable tissue? [1]. Although acquisition is more prompt than MRI, noncontrast CT typically provides less detailed information than DWI sequence on MRI [2]. According to the American Heart Association/American Stroke Association guidelines for early management in adults with ischemic stroke, "The prior guidelines recommended that CT be the primary diagnostic brain imaging study for the evaluation of patients with suspected stroke. Although CT is the 'criterion standard' with which other brain imaging studies are compared, it is relatively insenstitive in detecting acute and small cortical or subcortical infarctions, especially in the posterior fossa" [3, 4]. Multimodal MRI, although slightly more time consuming for protocol completion than multimodal CT, this imaging modality is able to display pristine images of the brain parenchyma within a short amount of minutes, and offers extremely sensitive detection and identification of new ischemic lesions that appear as hyperintensities of DWI and correlative Apparent Diffusion Coefficient (ADC) hypointensity, minutes from ictus in animal models [5]. In human studies, DWI has been reported to reveal infarcts sooner than conventional T2-weighted spinechocardiographic imaging [6]. Although contrast-induced nephrotoxicity may encumber multimodal CT or MRI, the use of gadolinium contrast may be somewhat more tolerable.
Although MRI is becoming more widely used, CT currently remains the principle imaging tool for stroke assessment in most stroke centers in the developed world. The existence of particular strengths and limitations of each methodology has lent itself to the use of a number of imaging techniques in combination to gather the most insight into the pathophysiology of underlying disease and to guide future therapy. We will discuss imaging modalities as they relate to mechanisms of disease in both intracerebral hemorrhage, as well as ischemic stroke resulting from small vessel, large vessel, or thromboembolic disease.

\section{Intracerebral Hemorrhage}

Etiologies of intracerebral hemorrhage vary widely but have traditionally been categorized as either likely related to cerebral amyloid angiopathy (CAA), or hypertension in primary intracerebral hemorrhage, or as a result of various other causes, under the terminology of secondary hemorrhage. However, these diagnoses are pathological diagnoses and cannot be defined with complete certainly with imaging alone. Another pattern of intracerebral hemorrhage, initially described in the 1990s [7-9] as microbleeds, has been shown from an imaging perspective in a number of publications, yet the data remains sparse for methodologically sound studies that describe precise pathophysiology underlying disease, and the implications it may carry in regard to patient management and stroke risk reduction [10]. To provide a more generalized view, intracerebral hemorrhage categories can also be considered as being either lobar in location or within the deep white matter. From an imaging perspective, etiologies leading to either primary or secondary intracerebral hemorrhage typically produce a different pattern or combination of patterns on CT and MRI, lending insight into the mechanism responsible for the bleeding. The use of allowing different modes of imaging modalities to not only diagnose, but to uncover the mechanism or etiology of hemorrhage and go on to guide treatment decisions and predict outcomes is formidable. In an emergency setting, both CT and MRI are able to easily and reliably detect intracerebral hemorrhage [11]. Subtle distinction in GRE signal intensities and patterns may discern underlying etiology of a hemorrhage, including hemorrhagic transformation of an ischemic infarct and primary hemorrhage (Fig. 3). Traditionally, the noncontrast CT scan has been believed of as the mainstay and first-line imaging modality for intracranial hemorrhage, also offering the ability to generate 3-dimensional volume estimates of blood accumulation [12]. Using imaging as an adjunctive resource throughout the clinical course of hemorrhage is integral as the prediction of hemorrhagic extension is often initially difficult to forsee [13]. Because therapeutic 

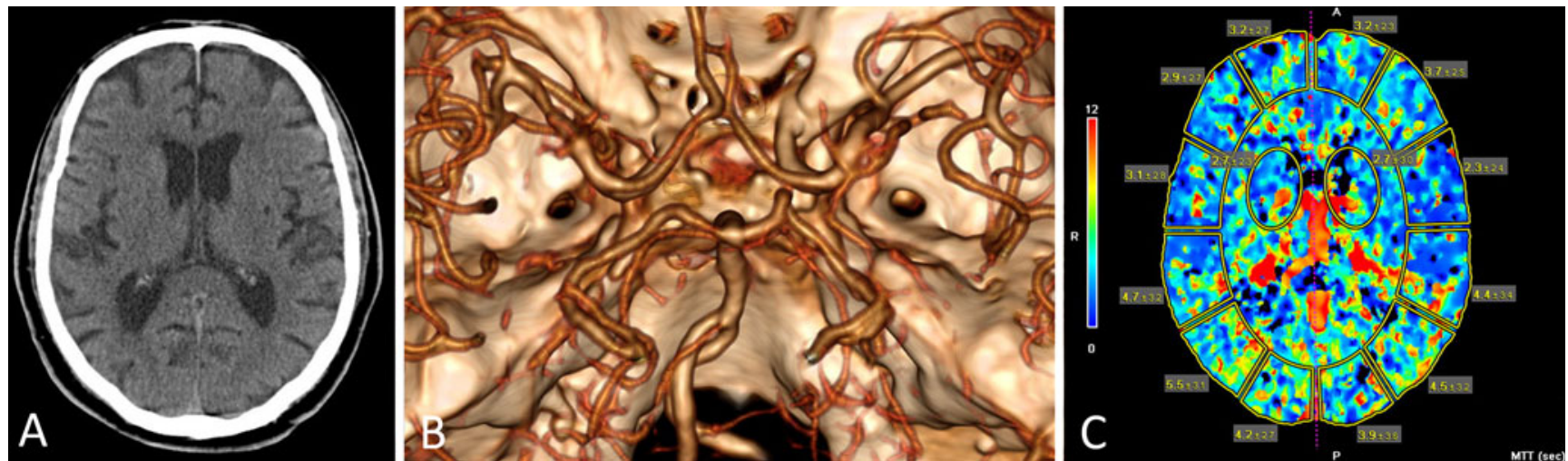

Fig. 1 Demonstration of multimodal computed tomographic (CT) acquisition, including (a) noncontrast CT, (b) CT angiography, and (c) CT perfusion. The patient is a 91-year-old man who presented with acute onset of slurred speech

management is so critically dependent on the interpretation of both the pathophysiological basis of hemorrhage as well as a prediction of suspected outcome, imaging surrogates foretelling hemorrhagic extension are vital [14]. One such foretelling sign of impending expansion is the nonhomogenous nature of the observed hematoma on CT (Fig. 4) [15].

Even though noncontrast CT alone offers some advantages in terms of wide availability, speed of image gathering and familiarity, further analysis for instance using multi- modal CT and CTA in combination offer a much broader picture, which lends itself to defining the pathophysiology of the bleeding. Although CTA will be discussed in much further detail in following articles, in brief, using this methodology of assessing vascular pathology in light of intracerebral hemorrhage may yield invaluable information in regard to the dynamic nature of bleeding in the tissue bed. Although noncontrast CT is the imaging procedure of choice during initial evaluation, the American Stroke
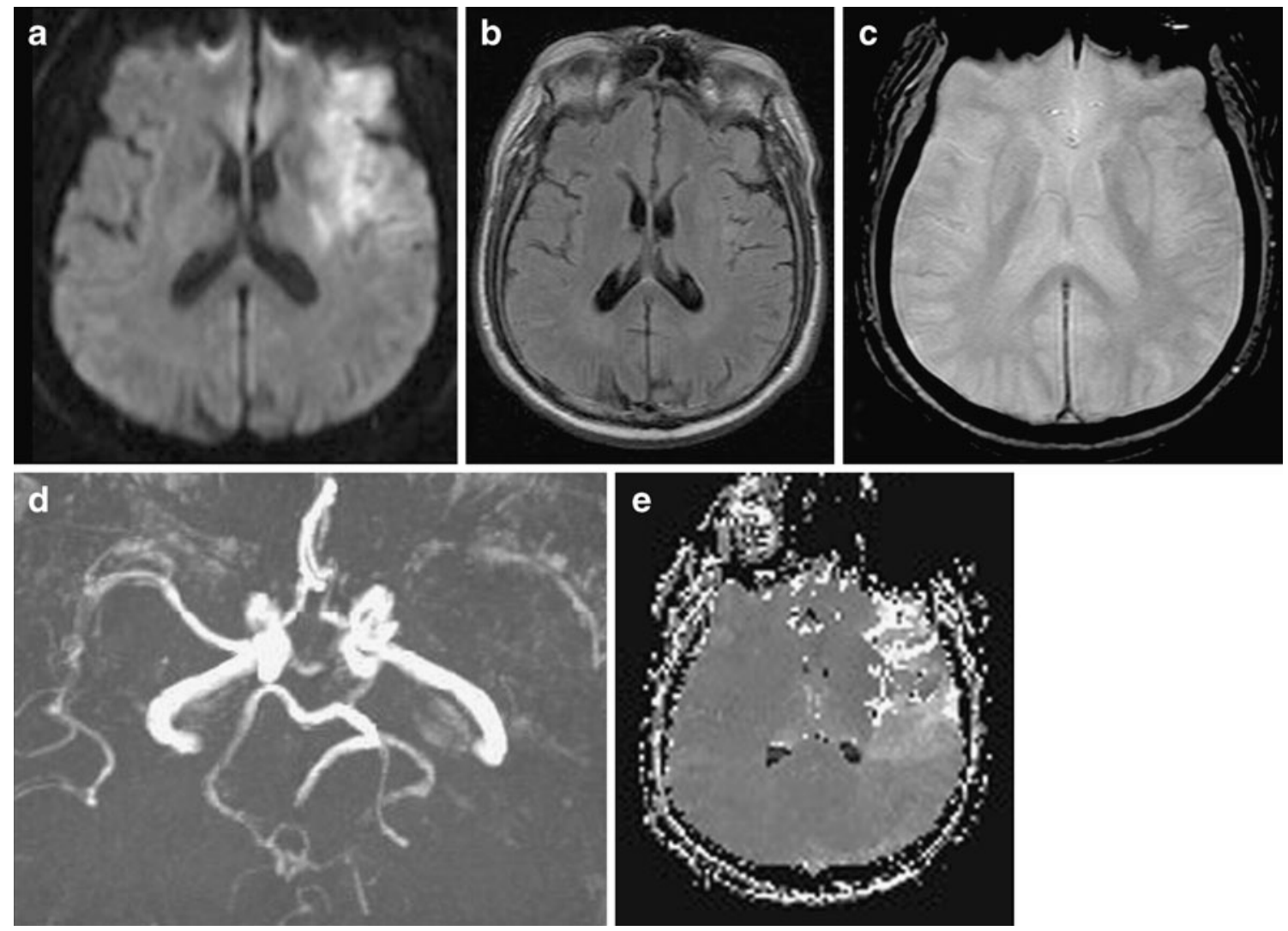

Fig. 2 Demonstration of multimodal magnetic resonance imaging acquisition, including (a) diffusion-weighted imaging, (b) fluidattenuated inversion recovery, (c) gradient recalled echocardiogram,

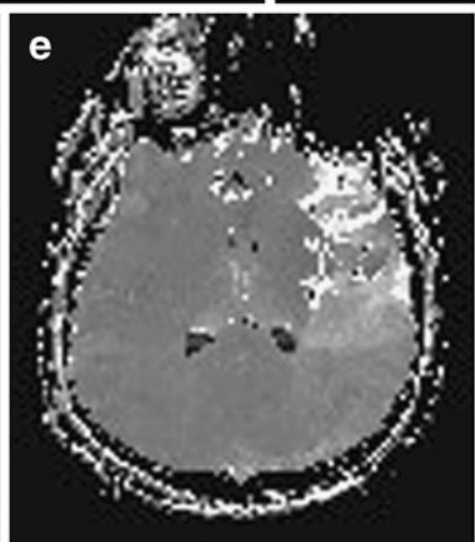

(d) magnetic resonance angiography of the head and neck, and (e) perfusion-weighted imaging. Patient is a 65-year-old man who presented with acute onset of right hemiplegia and global aphasia 
Fig. 3 Gradient recalled echocardiogrammagnetic resonance imaging sequences reveal subtle distinction in underlying etiology of hemorrhage. (a) Conflunent peticheal hemorrhagic transformation of ischemic infarct with central hypointensity in caudate and putamen and surrounding hyperintensity. (b) Slit-like cavity in the putamen with a surrounding rim of hypointensity revealing prior hemorrhage
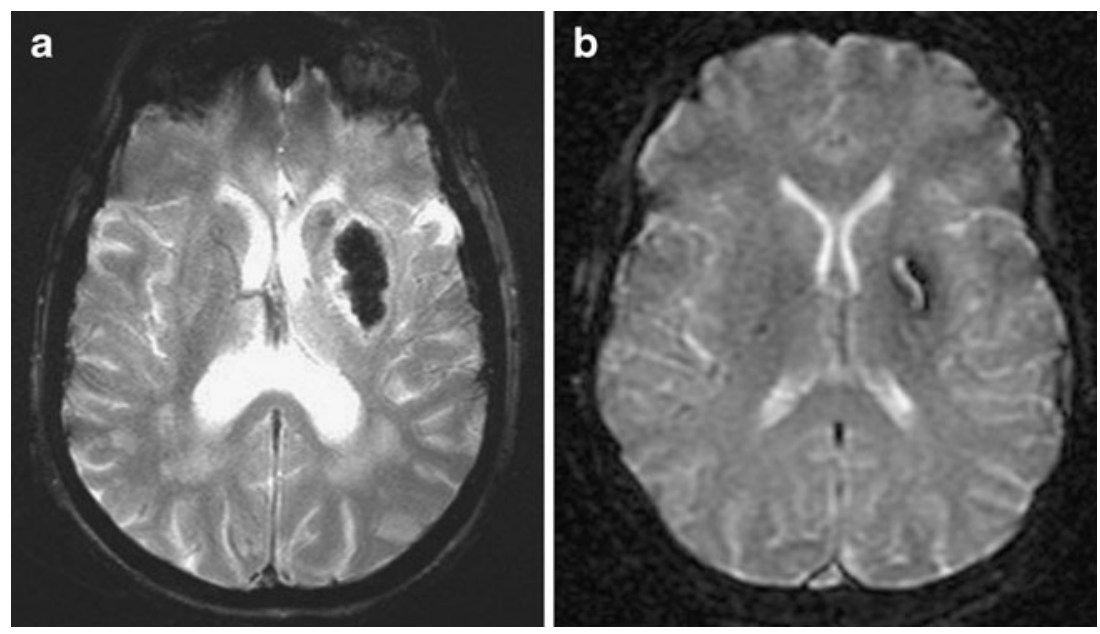

Association details allowing the clinical scenario to dictate further imaging investigations. For example, in the case of patients without clear cause of hemorrhage, who are young, normotensive, and clinically stable, angiography should be considered to investigate for possible structural lesions [16]. Also, Thompson et al. [17] in 2009 describe the CTA "spot sign" as "spot-like foci of enhancement within the margin of a parenchymal hematoma without connection to outside vessels...greater than $1.5 \mathrm{~mm}$ in maximal dimension and has a Hounsfield unit density at least double that of background hematoma density." This sign denotes active bleeding and is predictive of likely hemorrhagic extension. The key in identifying this sign is a direct comparison between CTA source images and the noncontrast CT. Typically this radiographic finding is believed to be specific in primary intracerebral hemorrhage, as in the cases of hypertensive bleed and CAA-related hemorrhage, yet more infrequently associated with secondary intracerebral hemorrhage [18]. With imaging identification of this sign, it is important to note that several vascular and nonvascular mimics exist, which include but are not limited to central nervous system malignancy, resulting in intraparenchymal hemorrhage $[18,19]$. The degree of extension of hemorrhage in itself may lie in the balance between intracranial pressure differentials, pressure differentials from adjacent tissue, and venous structures, as well as baseline condition of the surrounding white matter [20,21].

In terms of further detailed imaging, controversy has surrounded perihematomal surveillance in the days, and for better resolution, up to 1 week after hemorrhage [22]. Varying accounts of hemorrhage extension have surfaced, some describing alteration in the cerebral blood volume $(\mathrm{CBV})$ in the area of expansion using $\mathrm{CT}$ perfusion and others recounting the identification of penumbral territories, although perfusion measurements in these regions were often mild and perhaps not achieving true penumbral levels [23-25].Rather than frank ischemia, the most likely etiology for perihematomal tissue findings may be attributable to the effects of local compression or clot-mediated toxic factor release [25]. Using MRI imaging, Lou et al. [26] demonstrateda correlation between iron content in the area of edema and the degree of perihematomal edema.
Fig. 4 Computed tomographic scan demonstrating hemorrhagic extension of intracerebral hematoma for an 8-hour period of time. (a) Initial imaging and (b) 8-hr follow-up
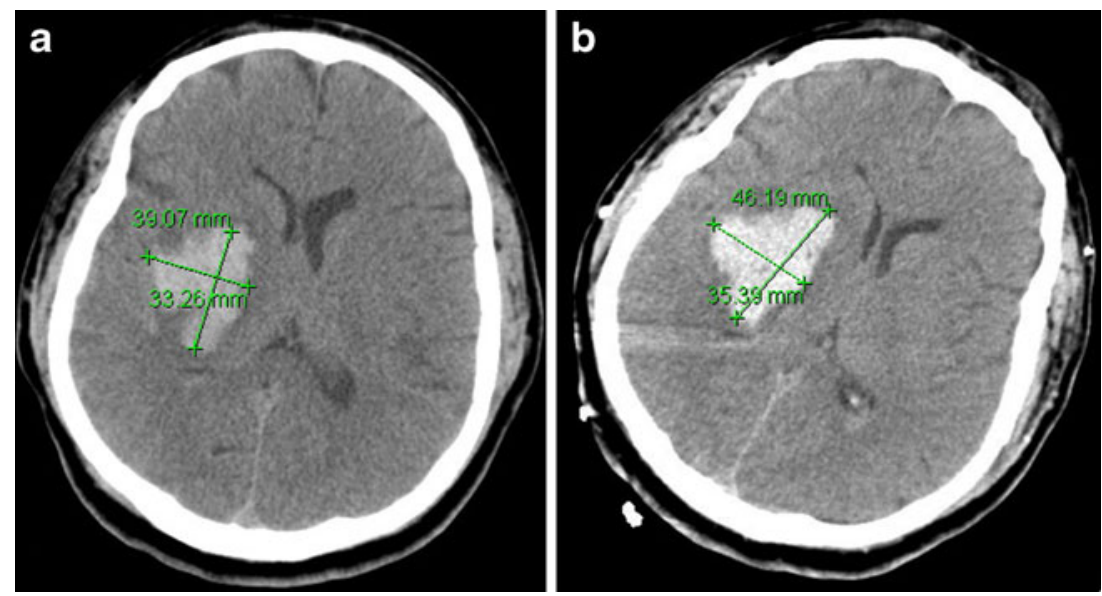
MRI certainly adds to the assessment of prognosis in terms of offering additional insight into potential secondary neuronal injury resultant from initial hematoma [20].

It is also important to note that in some cases evidence of blood may make itself apparent as a localized hematoma, although characteristically, primary intracerebral hemorrhage resultant from CAA and hypertension results in a pattern of either diffuse small vessels ischemic disease or small (less than or equal to 5-10 $\mathrm{mm}$ ) hypointense, punctate round parenchymal lesions on GRE MRI sequences.A number of variable imaging patterns and findings exist in secondary intracerebral hemorrhage, given the widely variable causes of hemorrhage. Underlying modifiers include conditions that affect vascular permeability and blood coagulation, and may concomitantly exist in the presence of vascular malformation, infection, or central nervous system malignancy.In some cases, hemorrhage may mask the underlying pathology on initial presentation as in the case of vascular malformations, and in other cases the hemorrhage itself directly lends clues into the existent mechanism of disease. As seen with coagulopathic conditions or in instances of venous hypertension, a blood-fluid level is usually a telling feature [20]. When taken together, this highlights the importance of multi-modal brain imaging, as well as detailed and dynamic angiographic imaging, because these modalities independently and in conjunction offer important insight into underlying pathophysiology of hemorrhage, and therefore dictate appropriate treatment.

Hemorrhagic transformation of ischemic stroke (Fig. 5) is one of the recognized subsets of intracerebral hemorrhage that has been confounded from an imaging standpoint. The difficulty lies in variability of distinguishing primary hemorrhagic processes from hemorrhagic infarction, as assessed by CT [27]. Although hemorrhagic transformation has been described as occurring independently, it has also been described in association with revascularization therapies [28]. Prediction of susceptibility to hemorrhagic transformation using imaging clues is crucial in treatment decision- making, particularly when it comes to choosing between revascularization therapy versus conservative, supportive management. Variables that are believed to contribute to the fate of hemorrhagic transformation after vascular occlusive stroke include metabolic alterations, such as hyperglycemia, reperfusion, surrounding tissue integrity, and of course, the severity of ischemia. In cases of hyperglycemia, the increased tendency for hemorrhagic transformation is believed to be related to an increase in extracellular glutamate and in intracellular acidosis, as well as progressive tissue edema [29]. Typically recognized imaging predictors of hemorrhage that parallel underlying pathology include low CBV, high burden of DWI hyperintense lesions on MRI, and accompanying hypointense ADC lesions [30-32]. Cho et al. [33] have also described an association between hemorrhagic transformation and the presence of focal fluid-attenuated inversion recovery hyperintensities within the regions of acute infarction. Several studies have now proposed another promising endpoint for assessment of risks for hemorrhage prior to the administration of revascularization therapy, namely permeability imaging as a surrogate marker of potential disturbances in blood brain barrier integrity [34-37]. In a prime example of how imaging may change the perception of pathophysiology and alter treatment strategies, evidence of blood brain barrier abnormalities on CT or MRI in the acute stage of ischemia may alter treatment to avoid intravenous tissue-plasminogen activator if the perceived elevated risk of hemorrhagic transformation is concerning in patients who would otherwise benefit from thrombolysis [38]. Nevertheless, although all of these studies are suggestive, in relation to clinical outcome, the extent of the ischemic penumbra, the influence of experimental treatments for ischemic stroke, and the effect of reperfusion have all yet to be fully evaluated.

Early imaging reassessment of tissue after reperfusion is often complicated by residual contrast from prior imaging. This contrast retention (Fig. 6) and engorgement of vasculature, most prominently described in the lenticulos-
Fig. 5 Hemorrhagic conversion in ischemic stroke. (a) Early evidence of ischemia on diffusion-weighted imaging in left middle cerebral artery occlusion followed by (b) hemorrhagic transformation and secondary mass effect on computed tomography
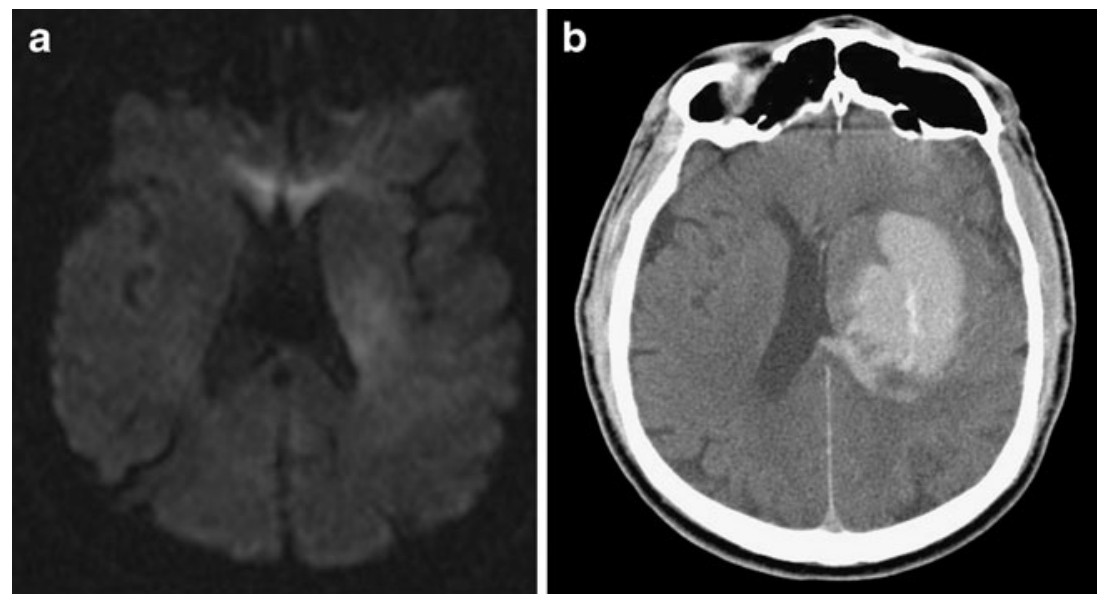


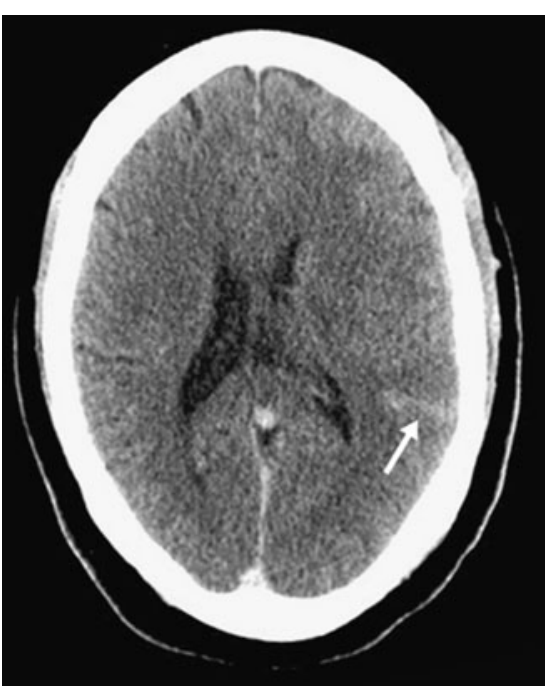

Fig. 6 Contrast retention (arrow) on follow-up computed tomographic scan after acute stroke

triate arteries from an imaging perspective may cause MRI T1-weighted hyperintensity and CT hyperdensity, and may be misleading in terms of potential for misrepresenting extension of a hemorrhagic focus.As the need for more precisely tailored imaging modalities to distinguish between contrast staining and intracerebral hemorrhage grows, more groups are experimenting with customized imaging modalities. Most recently, Gupta et al. [39] evaluated the efficacy of dual-energy $\mathrm{CT}$ in differentiating between iodinated contrast material staining and true hemorrhage, and concluded the high specificity and sensitivity of this imaging modality in delineating the relative contribution of each state to the apparent lesions.

\section{Small, Large Vessel, and Thromboembolic Disease Leading to Ischemic Stroke}

Although stroke is a largely heterogeneous disorder, spanning the years a number of classification systems have been generated to place this disease in categorized subtypes [40]. Each proposed system has offered its unique strengths and weaknesses. The major categories of disease leading to ischemic stroke can be subdivided into stroke secondary to small vessel, large vessel, thromboembolic disease, or as opposed to what some define as cryptogenic, and really any combination of the aforementioned etiologies. Even in ischemic stroke, the use of imaging modalities tailored for the diagnosis of stroke extend far beyond their diagnostic role to one of providing evidence for underlying mechanisms and implied treatment decisions.Vascular disease resulting in tissue ischemia is most often thought of as a direct result of hypoperfusion. This hypoperfusion can result from vascular stenosis in which case blood flow is reduced in a manner related to the degree of vascular compromise in an artery or vein, and can also occur as a result of complete vascular occlusion.Even in cases of complete occlusion, the radiographic finding of "tram tracking" (Fig. 7) [41], indicative of a degree of flow, albeit minimal, may offer favorable prognosis with spontaneous or therapeutic recanalization. Again, further extensive discussion of neck and cerebral angiography, although integral to the visualization of pathology, will be detailed in following articles. The resultant significance of vascular stenosis or occlusion on hypoperfusion and ischemia of the adjacent tissue bed is integrally related to the ability of collateral circulation to perfuse the underserved region [42].

In the setting of hyperacute ischemic stroke, noncontrast CT scanning has been, and still remains in some institutions, the first-line imaging modality. Although $\mathrm{CT}$ alone is notoriously known for its inability to detect early ischemic changes, in combination with its other subsets, multimodal $\mathrm{CT}$ in the setting on stroke evolution findings of perceived "ischemia" may simply reflect an increase in water content secondary to a response of increased $\mathrm{CBV}$, rather than pathological intracellular swelling [43, 44]. Parsons et al. [44], using noncontrast and perfusion CT, describe isolated focal swelling as correlated with penumbral tissue, whereas parenchymal hypo-attentuation as identifying the core infarct. The emergence of DWI [45-47], as an MRI imaging modality, has revolutionized the field of stroke imaging, especially in the hyperacute diagnosis, identification of underlying disease pattern (Fig. 8), therapeutic

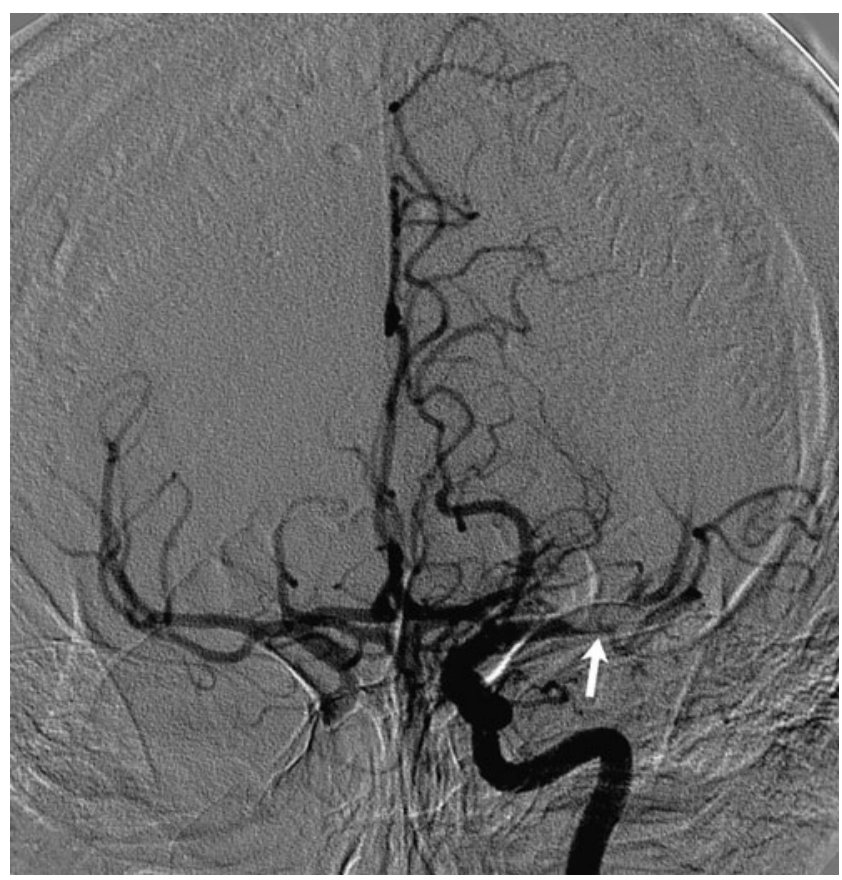

Fig. 7 Demonstration of tram track appearance on cerebral angiography in acute stroke showing contrast passage around the occlusive thrombus 

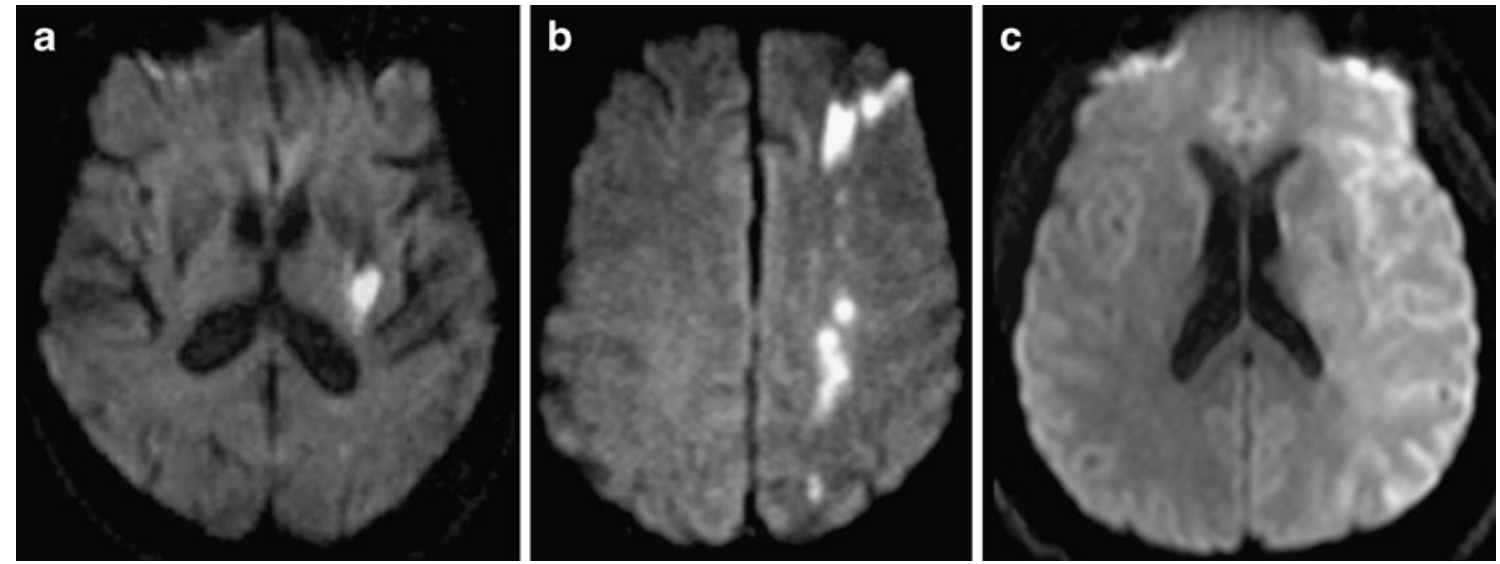

Fig. 8 Magnetic resonance imaging diffusion-weighted imaging comparison of radiographic lesion patterns resulting from different etiologies of ischemic stroke: (a) lacune, (b), borderzone infarct, and (c) full territorial infarction of the middle cerebral artery

management, and prediction of prognosis.DWI hyperintense lesions with ADC hypointense correlates are indicative of parenchymal cytotoxic edema, and their pattern of distribution is most informative with respect to likely underlying etiology of ischemic injury [48]. With the knowledge of arterial territories, punctate lesions for example, on one hand, may represent a thromboemoblic mechanism or alternatively simply indicate hypoperfusion with resultant borderzone ischemia in the vascular territory [49].

In terms of vascular pathology, atherosclerotic lesions leading to ischemic stroke can be readily identified using both multimodal CT and MRI. Even with noncontrast CT images, large vessel occlusion may be easily recognized by indirect features. What has been described as a "hyperdense" middle cerebral artery as a sign of vascular occlusion (Fig. 9), has now also been similarly described in the internal carotid and posterior cerebral arteries as well

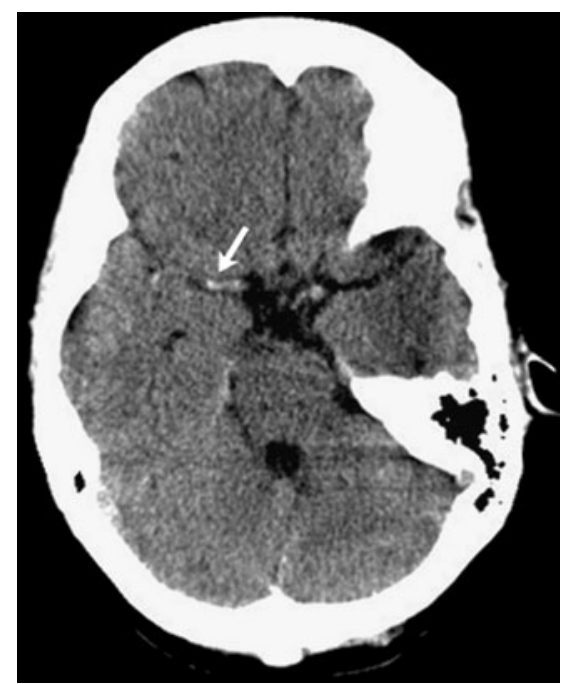

Fig. 9 Computed tomographichead scan demonstrating hyperdense middle cerebral artery sign (arrow)
[50, 51]. In terms of lesions associated with a clot, hyperdensities observed by CT imaging are often incorrectly attributed to tissue ischemia [52]. Analysis of clot composition in correlation with radiographic findings in both CT and GRE sequences on MRI has been particularly significant.It has been shown that in harvested human acute ischemic stroke clots that appeared hyperdense on CT scan and hypointense on GRE sequence, thrombi appeared to be rich in red cell content [53].

Brain imaging, including perfusion imaging, has become increasingly valuable in designing therapeutic strategies for ischemic stroke. Defining the ischemic core by CT or MRI is essential in identifying a pattern of the compromised vascular territory and its resultant extension.DWI lesions, for example, in the acute setting are believed to approximate the ischemic core, even though it is believed that there exists some potential reversibility to these lesions [51, 54, 55]. Because variable estimations of ischemic burden are generated with $\mathrm{CT}$ vs MRI imaging, taking perfusion imaging into account may provide the most accurate complementary view of tissue integrity and allow for prediction of tissue fate. Using contrast perfusion with gadolinium in the case of MRI and iodinated agents in the case of $\mathrm{CT}$, contrast labeling through the vasculature and their tissue beds is able to be reliably detect, and based on mapping and distribution, CBV and blood flow may be extrapolated. Arterial spin-labeled MRI perfusion (Fig. 10), in particular, has been shown to allow for determination of absolute measurements of cerebral blood flow [56-58]. These dynamic measurements compare normal parenchyma to patterns of perfusion in the ischemic core and in the surrounding penumbral regions. Perfusion-diffusion mismatch models with MRI compare the volume of hypoperfusion to the DWI hyperintense region.These perfusion map abnormalities in comparison to the ischemic core are commonly used as an estimate or gauge of mismatch 


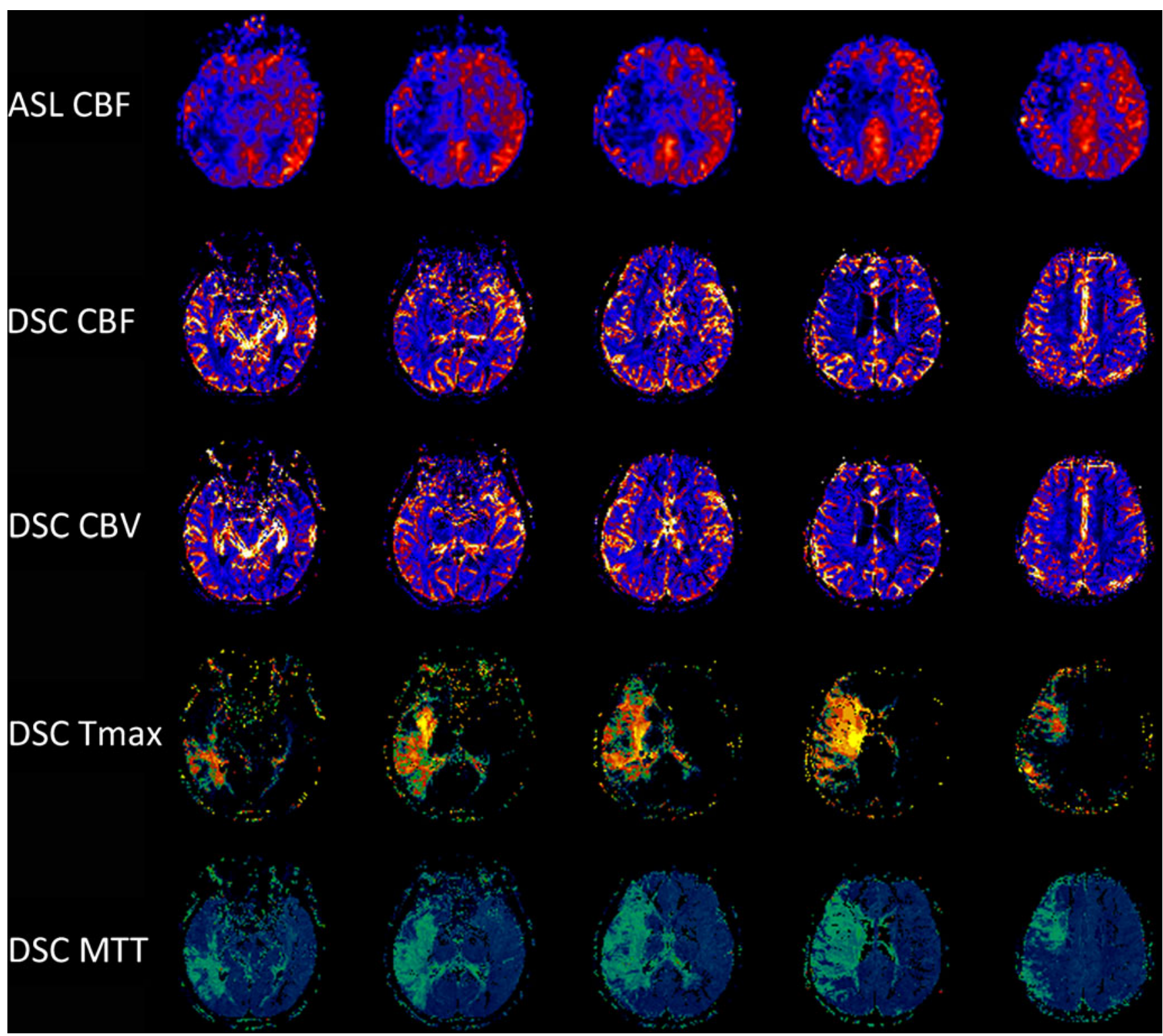

Fig. 10 Display of arterial spin label (ASL) MRI perfusion in acute right middle cerebral artery stroke. $\mathrm{CBF}=$ cerebral blood flow; $\mathrm{CBV}=\mathrm{cerebral}$ blood volume; DSC Tmax = dynamic susceptibility contrast, maximum temperature; DSC MTT = dynamic susceptibility contrast, mean transit time

between tissue severely affected by ischemia as in the ischemic core and the surrounding penumbral tissue that may benefit from therapeutic revascularization. In addition, although perfusion does offer information in regard to dynamic changes in the tissue bed, what it does not address is compensatory changes in vascular circulation, including the magnitude and pattern of development of arterial collaterals or venous drainage [52], which necessitates the complimentary use of angiography. However, in recent years unique postprocessing techniques have allowed for the construction of perfusion parameter maps from conventional angiography, allowing for simultaneous visualization of both relative perfusion, as well as vascular anatomical definition [59].
In terms of advancement in parenchymal imaging, several techniques in both the realm of CT and MRI have been developed to improve the sensitivity of imaging acquisition and postprocessing. One such example is the use of adaptive smoothing filters, as applied to a noncontrast CT scan to help improve the visualization of hypoattenuation in the early stages of ischemia [60]. Even with the ability to detect ischemic tissue damage within minutes of stroke onset, sodium MRI has been assessed for its potential to yet improve on earlier detection of tissue bed ischemia [61]. Techniques focusing on the potentially salvageable penumbra are equally as important with researchers demonstrating the ability to show potential microstructural damage within the tissue at risk [62]. In the midst of these advances, the 
need continues to exist for further refining of imaging modalities to allow for patient specific tailoring of therapy and improvement in clinical outcome.

\section{Summary}

Imaging of the brain in stroke with $\mathrm{CT}$ and MRI techniques has become an invaluable tool not only in the initial diagnosis of stroke, but in clarifying the mechanisms of ischemia or hemorrhage in the brain. Elucidating these mechanisms has direct implications on expected prognoses and therapeutic interventions aimed at altering the course of the disease. The ability to generate a time efficient and reliable understanding of stroke etiology with imaging is essential in guiding treatment options to improve clinical outcomes. Not only is such imaging crucial in the hyperacute setting, but it is also crucial in the setting of evolving stroke pathology, tailoring each therapeutic decision based on imaging prediction of progression and likely prognosis. The refinement in imaging modalities must ultimately provide the clinical community with methods that can be widely available, efficient, costeffective, and reproducibly interpretable, posing a continuous challenge for the upcoming years.

\section{Citation Methodology}

The literature search was started on October 10, 2010 and was updated through March 29, 2011 following reviewer comments. PubMed was searched through the website at http:// www.ncbi.nlm.nih.gov/pubmed/, with no restrictions in terms of date ranges. A number of keywords were used including: ischemic stroke, hemorrhagic stroke, MRI, CT, multimodal MRI, multimodal CT, microbleeds, intracranial hemorrhage, DWI, American Stroke Association guidelines, pneumbra, hyperglycemia and stroke, thromboembolic disease, vascular occlusion, and reperfusion. The literature searchwas supplemented by a review of key journals, including, but not limited to Stroke, Brain, Lancet, and Neurology, as well as the authors' previously cited article database file. Cited articles were read, reviewed in full, and discussed by the coauthors.

Acknowledgment Full conflict of interest disclosure is available in the electronic supplementary material for this article.

\section{References}

1. Rastogi SaLD. Multimodal computed tomography in acute ischemic stroke. Brain Trauma Stroke 2010:50-54.

2. Selco SL, Liebeskind DS. Hyperacute imaging of ischemic stroke: role in therapeutic management. Curr Cardiol Rep 2005;7:10-15.
3. Mullins ME, Schaefer PW, Sorensen AG, et al. CT and conventional and diffusion-weighted MR imaging in acute stroke: study in 691 patients at presentation to the emergency department. Radiology 2002;224:353-360.

4. Adams HP Jr., del Zoppo G, Alberts MJ, et al. Guidelines for the early management of adults with ischemic stroke: a guideline from the American Heart Association/American Stroke Association Stroke Council, Clinical Cardiology Council, Cardiovascular Radiology and Intervention Council, and the Atherosclerotic Peripheral Vascular Disease and Quality of Care Outcomes in Research Interdisciplinary Working Groups: the American Academy of Neurology affirms the value of this guideline as an educational tool for neurologists. Stroke 2007;38:1655-1711.

5. Rother J, de Crespigny AJ, D'Arceuil H, Mosley ME. MR detection of cortical spreading depression immediately after focal ischemia in the rat. J Cereb Blood Flow Metab 1996;16:214-220.

6. Warach S, Chien D, Li W, Ronthal M, Edelman RR. Fast magnetic resonance diffusion-weighted imaging of acute human stroke. Neurology 1992;42:1717-1723.

7. Chan S, Kartha K, Yoon SS, Desmond DW, Hilal SK. Multifocal hypointense cerebral lesions on gradient-echo MR are associated with chronic hypertension. AJNR Am J Neuroradiol 1996; 17:18211827.

8. Greenberg SM, Finklestein SP, Schaefer PW. Petechial hemorrhages accompanying lobar hemorrhage: detection by gradientecho MRI. Neurology 1996;46:1751-1754.

9. Offenbacher H, Fazekas F, Schmidt R, et al. MR of cerebral abnormalities concomitant with primary intracerebral hematomas. AJNR Am J Neuroradiol 1996;17:573-578.

10. Cordonnier C, Al-Shahi Salman R, Wardlaw J. Spontaneous brain microbleeds: systematic review, subgroup analyses and standards for study design and reporting. Brain 2007;130:1988-2003.

11. Chalela JA, Kidwell CS, Nentwich LM, et al. Magnetic resonance imaging and computed tomography in emergency assessment of patients with suspected acute stroke: a prospective comparison. Lancet 2007;369:293-298.

12. Strik HM, Borchert H, Fels C, et al. Three-dimensional reconstruction and volumetry of intracranial haemorrhage and its mass effect. Neuroradiology 2005;47:417-424.

13. Broderick JP, Diringer MN, Hill MD, et al. Determinants of intracerebral hemorrhage growth: an exploratory analysis. Stroke 2007;38:1072-1075.

14. Anderson CS. Medical management of acute intracerebral hemorrhage. Curr Opin Crit Care 2009;15:93-98.

15. Ji N, Lu JJ, Zhao YL, Wang S, Zhao JZ. Imaging and clinical prognostic indicators for early hematoma enlargement after spontaneous intracerebral hemorrhage. Neurol Res 2009;31:362-366.

16. Broderick JP, Adams HP, Jr., Barsan W, et al. Guidelines for the management of spontaneous intracerebral hemorrhage: A statement for healthcare professionals from a special writing group of the Stroke Council, American Heart Association. Stroke 1999;30:905915.

17. Thompson AL, Kosior JC, Gladstone DJ, et al. Defining the CT angiography 'spot sign' in primary intracerebral hemorrhage. Can J Neurol Sci 2009;36:456-461.

18. Gazzola S, Aviv RI, Gladstone DJ, et al. Vascular and nonvascular mimics of the CT angiography "spot sign" in patients with secondary intracerebral hemorrhage. Stroke 2008;39:1177-1183.

19. Yoon DY, Chang SK, Choi CS, Kim WK, Lee JH. Multidetector row CT angiography in spontaneous lobar intracerebral hemorrhage: a prospective comparison with conventional angiography. AJNR Am J Neuroradiol 2009;30:962-967.

20. Liebeskind DS. Imaging the future of stroke: II. Hemorrhage. Ann Neurol 2010;68:581-592.

21. Lou M, Al-Hazzani A, Goddeau RP Jr., Novak V, Selim M. Relationship between white-matter hyperintensities and hematoma 
volume and growth in patients with intracerebral hemorrhage. Stroke 2010;41:34-40.

22. Pascual AM, Lopez-Mut JV, Benlloch V, et al. Perfusion-weighted magnetic resonance imaging in acute intracerebral hemorrhage at baseline and during the 1st and 2nd week: a longitudinal study. Cerebrovasc Dis 2007;23:6-13.

23. Fainardi E, Borrelli M, Saletti A, et al. CT perfusion mapping of hemodynamic disturbances associated to acute spontaneous intracerebral hemorrhage. Neuroradiology 2008;50:729-740.

24. Fainardi E, Borrelli M, Saletti A, et al. Evaluation of acute perihematomal regional apparent diffusion coefficient abnormalities by diffusion-weighted imaging. Acta Neurochir Suppl 2006;96:81-84.

25. Gass A. Is there a penumbra surrounding intracerebral hemorrhage? Cerebrovasc Dis 2007;23:4-5.

26. Lou M, Lieb K, Selim M. The relationship between hematoma iron content and perihematoma edema: an MRI study. Cerebrovasc Dis 2009;27:266-271.

27. Lovelock CE, Anslow P, Molyneux AJ, et al. Substantial observer variability in the differentiation between primary intracerebral hemorrhage and hemorrhagic transformation of infarction on CT brain imaging. Stroke 2009;40:3763-3767.

28. Khatri P, Wechsler LR, Broderick JP. Intracranial hemorrhage associated with revascularization therapies. Stroke 2007;38:431-440.

29. Gilmore RM, Stead LG. The role of hyperglycemia in acute ischemic stroke. Neurocrit Care 2006;5:153-158.

30. Campbell BC, Christensen S, Butcher KS, et al. Regional very low cerebral blood volume predicts hemorrhagic transformation better than diffusion-weighted imaging volume and thresholded apparent diffusion coefficient in acute ischemic stroke. Stroke 2010;41:82-88.

31. Lee SH, Kim BJ, Ryu WS, et al. White matter lesions and poor outcome after intracerebral hemorrhage: a nationwide cohort study. Neurology 2010;74:1502-1510.

32. Singer OC, Humpich MC, Fiehler J, et al. Risk for symptomatic intracerebral hemorrhage after thrombolysis assessed by diffusionweighted magnetic resonance imaging. Ann Neurol 2008;63:5260.

33. Cho AH, Kim JS, Kim SJ, et al. Focal fluid-attenuated inversion recovery hyperintensity within acute diffusion-weighted imaging lesions is associated with symptomatic intracerebral hemorrhage after thrombolysis. Stroke 2008;39:3424-3426.

34. Aviv RI, d'Esterre CD, Murphy BD, et al. Hemorrhagic transformation of ischemic stroke: prediction with $\mathrm{CT}$ perfusion. Radiology 2009;250:867-877.

35. Fiehler J, Remmele C, Kucinski T, et al. Reperfusion after severe local perfusion deficit precedes hemorrhagic transformation: an MRI study in acute stroke patients. Cerebrovasc Dis 2005;19:117124.

36. Bang OY, Saver JL, Alger JR, et al. Patterns and predictors of blood-brain barrier permeability derangements in acute ischemic stroke. Stroke 2009;40:454-461.

37. Hom J, Dankbaar JW, Soares BP, et al. Blood-Brain Barrier Permeability Assessed by Perfusion CT Predicts Symptomatic Hemorrhagic Transformation and Malignant Edema in Acute Ischemic Stroke. AJNR Am J Neuroradiol 2011;32(1):41-48.

38. Kassner A, Roberts TP, Moran B, Silver FL, Mikulis DJ. Recombinant tissue plasminogen activator increases blood-brain barrier disruption in acute ischemic stroke: an MR imaging permeability study. AJNR Am J Neuroradiol 2009;30:1864-1869.

39. Gupta R, Phan CM, Leidecker C, et al. Evaluation of dual-energy CT for differentiating intracerebral hemorrhage from iodinated contrast material staining. Radiology 2010;257:205-211.

40. Amarenco P, Bogousslavsky J, Caplan LR, Donnan GA, Hennerici MG. Classification of stroke subtypes. Cerebrovasc Dis 2009;27:493501.
41. Otsuka Y, Waki R, Yamauchi H, et al. Angiographic demarcation of an occlusive lesion may predict recanalization after intraarterial thrombolysis in patients with acute middle cerebral artery occlusion. J Neuroimaging 2008;18:422-427.

42. Liebeskind DS. Collaterals in acute stroke: beyond the clot. Neuroimaging Clin N Am 2005;15:553-573.

43. Butcher KS, Lee SB, Parsons MW, et al. Differential prognosis of isolated cortical swelling and hypoattenuation on CT in acute stroke. Stroke 2007;38:941-947.

44. Parsons MW, Pepper EM, Bateman GA, Wang Y, Levi CR. Identification of the penumbra and infarct core on hyperacute noncontrast and perfusion CT. Neurology 2007;68:730-736.

45. Le Bihan D, Breton E, Lallemand D, et al. Separation of diffusion and perfusion in intravoxel incoherent motion MR imaging. Radiology 1988;168:497-505.

46. Moseley ME, Kucharczyk J, Mintorovitch J, et al. Diffusionweighted MR imaging of acute stroke: correlation with T2weighted and magnetic susceptibility-enhanced MR imaging in cats. AJNR Am J Neuroradiol 1990;11:423-429.

47. Moseley ME, Cohen Y, Mintorovitch J, et al. Early detection of regional cerebral ischemia in cats: comparison of diffusion- and T2-weighted MRI and spectroscopy. Magn Reson Med 1990;14:330-346.

48. Bang OY, Lee PH, Heo KG, et al. Specific DWI lesion patterns predict prognosis after acute ischaemic stroke within the MCA territory. J Neurol Neurosurg Psychiatry 2005;76:1222-1228.

49. Caplan LR, Wong KS, Gao S, Hennerici MG. Is hypoperfusion an important cause of strokes? If so, how? Cerebrovasc Dis 2006;21:145-153.

50. Krings T, Noelchen D, Mull M, et al. The hyperdense posterior cerebral artery sign: a computed tomography marker of acute ischemia in the posterior cerebral artery territory. Stroke 2006;37:399-403.

51. Ozdemir O, Leung A, Bussiere M, Hachinski V, Pelz D. Hyperdense internal carotid artery sign: a CT sign of acute ischemia. Stroke 2008;39:2011-2016.

52. Liebeskind DS. Imaging the future of stroke: I. Ischemia. Ann Neurol 2009;66:574-590.

53. Liebeskind D, Yong WH, Sanossian N, et al. CT and MRI early vessel signs reflect clot composition in acute stroke. Stroke 2008;39:564.

54. Kidwell CS, Saver JL, Mattiello J, et al. Thrombolytic reversal of acute human cerebral ischemic injury shown by diffusion/perfusion magnetic resonance imaging. Ann Neurol 2000;47:462-469.

55. Fiehler J, Foth M, Kucinski T, et al. Severe ADC decreases do not predict irreversible tissue damage in humans. Stroke 2002;33:79-86.

56. Chalela JA, Alsop DC, Gonzalez-Atavales JB, et al. Magnetic resonance perfusion imaging in acute ischemic stroke using continuous arterial spin labeling. Stroke 2000;31:680-687.

57. Golay X, Hendrikse J, Lim TC. Perfusion imaging using arterial spin labeling. Top Magn Reson Imaging 2004;15:10-27.

58. Hendrikse J, van der Grond J, Lu H, van Zijl PC, Golay X. Flow territory mapping of the cerebral arteries with regional perfusion MRI. Stroke 2004;35:882-887.

59. Liebeskind DS SG, Black SE, Buck BH. Perfusion angiography: a novel technique for the characterization of perfusion in cerebral ischemia. . Stroke 2008;39:576.

60. Takahashi N, Lee Y, Tsai DY, et al. Improvement of detection of hypoattenuation in acute ischemic stroke in unenhanced computed tomography using an adaptive smoothing filter. Acta Radiol 2008;49:816-826.

61. Jones SC, Kharlamov A, Yanovski B, et al. Stroke onset time using sodium MRI in rat focal cerebral ischemia. Stroke 2006;37:883-888.

62. Kosior RK, Wright CJ, Kosior JC, et al. 3-Tesla versus 1.5-Tesla magnetic resonance diffusion and perfusion imaging in hyperacute ischemic stroke. Cerebrovasc Dis 2007;24:361-368. 